\title{
NAFTA, la polémica y sus lecciones
}

\author{
Luis de Sebastián*
}

Resumen

El objeto de este artículo es mostrar que el resultado final del NAFTA, en cuanto enfrentamiento dialéctico, que refleja enfrentamientos de intereses económicos concretos, tiene una gran importancia para la configuración del orden comercial internacional en los próximos años. Asimismo, subraya que la novedad de NAFTA radica en ser un experimento en integración de economias de muy distinto nivel de desarrollo. La operación es en sí misma difícil y compleja por los muchos aspectos que tiene, pero es importante para articular un orden económico no excluyente. Es un experimento nuevo de integración norte-sur.

\section{Introducción: la polémica actual}

Pocas cosas, si exceptuamos el nuevo plan de salud de Hillary Clinton, son tan debatidas actualmente en Estados Unidos como el Acuerdo sobre el area de comercio libre en Norte América (NAFTA), del inglés North American Free Trade Area. El acuerdo, firmado por los presidentes de Estados Unidos, Canadá y México el 17 de diciembre de 1992, no ha sido ratificado todavia por el Congreso de Estados Unidos ${ }^{1}$ y puede que no sea ratificado nunca si vencieran las fuerzas que se han desatado contra él. En México también hay fuerzas políticas y sociales que se oponen al NAFTA, pero allí no es probable que éstas sean capaces de detener al rodillo del PRI.
El público europeo, embebido en sus propios -y no despreciables- problemas económicos, observa desde lejos y como cosa que no va con él las discusiones americanas. El objeto de este artículo es mostrar que esta percepción no es correcta; que, por el contrario, el resultado final de los enfrentamientos dialécticos, que reflejan enfrentamientos de intereses económicos concretos, tiene una importancia vital para la configuración del orden comercial internacional en los próximos años ${ }^{2}$.

\section{2. ¿Qué es el acuerdo NAFTA?}

Después de una década de intensas y a veces encontradas negociaciones comerciales, Estados

* Profesor de ESADE, Barcelona, y consultor del Banco Interamericano de Desartollo. Las opiniones son estrictamente personales y no reflejan el pensamiento de ninguna de las dos instituciones. 


\section{Se trata de ventilar las enormes quejas de los sindicatos sobre cosas que están sucediendo en Estados Unidos y las que van a suceder al margen del tratado de comercio con México.}

Unidos y México acordaron en 1990 iniciar un proceso tendiente a firmar un acuerdo de libre comercio ${ }^{3}$. En febrero de 1991, Canadá, Estados Unidos (que ya tenían un acuerdo de libre comercio entre ellos) y México comenzaron a negociar el NAFTA. Ese mismo año, el Congreso estadounidense aprobó un procedimiento de "vía rápida" que facultaba al presidente a negociar acuerdos comerciales sin tener que recurrir a aquél. El 12 de agosto de 1992, las tres partes dieron a conocerse que se habían llegado a un acuerdo sobre el contenido del tratado. El tipo de acuerdo al que llegaron es lo que técnicamente se conoce como "zona de libre comercio", que es una institución menos fuerte que un "mercado común", y se distingue de éste porque no contempla el establecimiento de un arancel exterior común. El Congreso no trató el tema en 1992 como se esperaba y ha dejado la discusión y el voto para finales de 1993. Por eso, la campaña a favor y en contra se ha acelerado en estos últimos meses del año.

3. La polémica: los demócratas rebeldes y Ross Perot

El NAFTA es una creatura de los republicanos que los demócratas tienen que llevar a cabo para complacer a su presidente. Pero algunos demócratas no quieren cargar con la herencia, no tanto por razones generales de línea de partido - que eso en Estados Unidos no existe- cuanto por su compromiso con la defensa de intereses particulares y locales. Se ha dado una verdadera sublevación en el campo demócrata. Los principales opositores al tratado, que ya han anunciado un voto negativo, son los representantes David E. Bonior, secretario ("látigo" en la terminología anglosajona) de la mayoría parlamentaria demócrata, y Richard Gephartdt, el líder de esta mayoría. Bonior, un demócrata de pro que votó invariablemente contra la política de Reagan en Nicaragua, representa al distrito obrero de Michigan, un estado que fabrica automóviles y odia al NAFTA. Gephardt, que tiene aspiraciones presidenciales, no quiere enfrentarse con los aparatos sindicales que controlan parte del voto demócrata y se oponen frontalmente al tratado. También se opone el representante de Massachusetts Barney Frank, otro de los hombres de los sindicatos en el Congreso, por miedo a que los obreros peor pagados salgan perdiendo4.

Estos demócratas, que como Bonior han sido de lo más "liberal" ("de izquierdas", en el argot político norteamericano), forman filas contra el NAFTA con fuerzas conservadoras como Pat Buchanan, político y publicista de ultraderecha, que mantiene una posición de completo aislacionismo (America First) y Ross Perot, el ex candidato presidencial, que sigue apelando a los sentimientos más elementales de los ciudadanos estadounidenses.

Su libro Salva tu puesto de trabajo, salva al país ${ }^{5}$, escrito con (o más bien por) el economista Pat Choate, es hoy por hoy la principal arma arrojadiza que tienen los opositores del NAFTA contra el encanto y el poder de convencer de Clinton. Los argumentos de Perot son medias verdades, como ahora veremos, que son las más fáciles de manejar, porque impactan con lo que tienen de verdad, sin tener el rigor que exigen las verdades enteras.

El argumento central de Perot es que los trabajadores estadounidenses, mejor entrenados, mejor pagados, protegidos por sindicatos libres y por una legislación social avanzada no pueden competir con los obreros mal pagados, sin sindicatos libres, sin protección ni seguridad social de México. Las diferencias de costos entre los dos países se deberían a la diferencia en las condiciones sociales de sus trabajadores (costos laborales, en definitiva). De esa manera, argumenta Perot, una situación más democrática y social es penalizada por el NAFTA para favorecer otra situación menos democrática y menos social. Es un nuevo argumento para el proteccionismo comercial: la protección contra el dumping social. Por cierto que el argumento podría usarse en la Comunidad Europea 


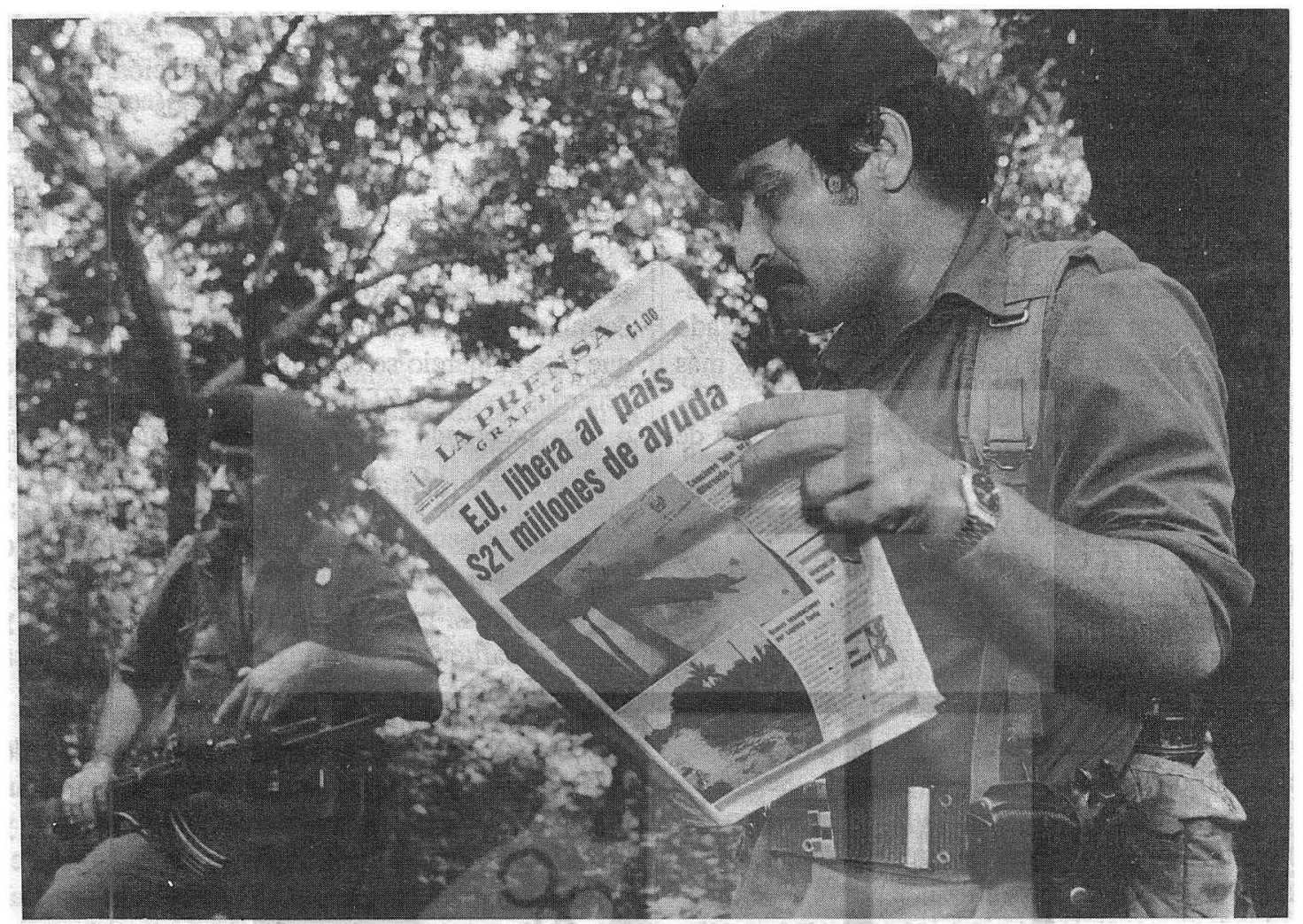

contra los productos estadounidenses que los producen obreros menos protegidos socialmente, y a veces peor pagados que los obreros europeos. Y llevado a sus últimas consecuencias, este argumento acabaría con todo el comercio de los países ricos con los países en desarrollo, donde las condiciones sociales de los trabajadores son más precarias que en los países industrializados. Buscar una igualdad de condiciones sociales como condición previa al comercio internacional sería una insensatez.

\section{Los argumentos ecologistas}

Contra el NAFTA también milita la mayor parte de las organizaciones ecologistas de Estados Unidos $^{6}$. Los argumentos de los ecologistas calaron fuerte y el gobierno de Washington se vio obligado a negociar unos acuerdos suplementarios para evitar que los diferentes estándares de protección ambiental y de la salud que se dan en México por una parte y Canadá y Estados Unidos por otra se vieran reducidos al mínimo común denominador, es decir, al de México.

Sin embargo, las provisiones que contienen los acuerdos suplementarios al tratado no parecen satisfacer a los ecologistas que siguen defendiendo sus argumentos contra el NAFTA. Se les ha dado dos tipos de respuestas. En primer lugar, recalcar que con el NAFTA los países siguen decidiendo qué niveles de protección del medio ambiente y de la salud de los ciudadanos van a regir en sus res pectivas jurisdicciones, es decir, negar que se vaya a dar un mínimo común 7 , y por otra parte, ayudar, por medio del Banco Mundial y otras instituciones internacionales, a elevar los niveles de sanitación y ecológicos de las regiones fronterizas de México con Estados Unidos.

5. El argumento de los sindicatos: la fuga de empresas

No es Ross Perot la fuerza central en la campaña para matar NAFTA, sino el movimiento sin- 
dical. Perot tiene pocos partidarios en el Congreso, pero los sindicatos han estado trabajando ferozmente para alinear a sus amigos y a los beneficiarios de la campaña contra el acuerdos.

Thomas Donahue, el tesorero de la poderosa federación sindical $A F L-C I O$, testificando ante el comité de finanzas del senado, en septiembre pasado, expuso los argumentos de los sindicatos. Muchas empresas estadounidenses se han desplazado a México buscando el trabajo barato, muchas más se van a ir, y NAFTA no ofreceria "ninguna protección contra una ulterior desindustrialización de la economía americana".

La cuestión verdadera sería: si fuera derrotado el acuerdo en el Congreso, ¿las empresas estadounidenses dejarían de irse a México o a otros paises de salarios más bajos? O la que preguntó al sindicalista Donahue el senador demócrata de Montana Max Baucus: ¿estarían mejor los obreros norteamericanos con el acuerdo que sin él? El senador no obtuvo una respuesta concreta. Según los partidarios de NAFTA, los sindicatos parecen ver el voto sobre él en el Congreso principalmente como una declaración simbólica sobre la moralidad o la inmoralidad de que las empresas se establezcan fuera del país (nada menos que la moralidad de la inversión directa en el extranjero) y sobre el comercio con países que tienen niveles de salarios inferiores. Mucho de este debate no es realmente sobre el acuerdo mismo y lo que va a o no va a hacer. Se trata de ventilar las enormes quejas de los sindicatos sobre cosas que están sucediendo en Estados Unidos y las que van a suceder al margen del tratado de comercio con México.

Probablemente, la oposición de los sindicatos a NAFTA recoge las angustias que los trabajadores sienten en unos momentos en que las grandes empresas están haciendo amplias reorganizaciones y despidos masivos para aumentar la eficiencia (la $I B M$, por ejemplo, va a despedir 60,000 empleados en cuatro años). Robert Reich, el Ministro de Trabajo, se refería a este fenómeno cuando dijo al mismo comité:

Creo que NAFTA es un elemento muy pequeño en comparación con la enorme ola de cambios que se abate sobre Estados Unidos'.

Cuatro quintas partes de la población mundial vive en economías con salarios bajos y allí están todos los mercados que crecen más rápidamente (China, India, Indonesia, Tailandia, etc.). El comercio estadounidense con el mundo en desarrollo y con México en particular va a seguir creciendo pase lo que pase con NAFTA. Lo que importa en definitiva es si será mejor para los estadounidenses que este comercio se desarrolle bajo las reglas que acuerdos como NAFTA establezcan deliberadamente - siga creciendo por iniciativas privadas desordenadamente. Los que están a favor de NAFTA piensan que, sin ninguna duda, será mejor para Estados Unidos. Esta respuesta, naturalmente, no responde a la cuestión de si esos "acuerdos ordenadores" serán mejor para el mundo en general y para los participantes de menor fuerza económica en particular.

Pero los sindicatos, asustados por los cierres de fábricas y por el descenso de la afiliación, están presionando fuerte a todos sus viejos amigos y aliados para que vengan en su ayuda ${ }^{10}$. El proteccionismo, sin embargo, por si sólo no puede crear empleados -aunque puede frenar su destrucción-, y Estados Unidos necesita un millón adicional de puestos de trabajo cada año, únicamente para hacer frente al crecimiento de la población. El Ministro Reich describió NAFTA, creo que correctamente, como un paso más, junto a otros que este pais ha dado en la década pasada para construir un régimen comercial que fortalezca el crecimiento económico de Estados Unidos de América sobre todo.

La clientela de los sindicatos está confinada a los límites de la economía nacional y sus políticas comerciales tienden al proteccionismo casi por definición. El factor trabajo es siempre nacional. Pero en esta polémica han aparecido en los sindicatos aclitudes exclusivistas anti tercer mundo. Por ejemplo, el mismo Lane Kirkland propuso que si fracasara el NAFTA - lo cual él está intentando con todas sus fuerzas-, el gobiemo debiera considerar la creación de un tratado del comercio libre del Atlántico Norte con Estados Unidos, Canadá y la Comunidad Europea, que incluiría casi la mitad 
de los productos nacionales combinados de todo el mundo. Este nuevo NAFTA (North Atlantic Free Trade Area) no incluiría a México, mientras no mejore las condiciones de vida de sus trabajadores. Kirland propone que se trate a México como a España y Portugal que, según este líder sindical, estuvieron excluidos de la Comunidad Europea hasta que mejoraron sus niveles de vida y las condiciones de trabajo.

Los críticos estadounidenses de NAFTA recurren a la comparación de la dimensión social en la legislación comunitaria (el tratado de Roma, el Acta única, el tratado de Maastricht) y el NAFTA.

NAFTA carece de las salvaguardias embrionales que contempla la CEE: no tiende fondos de desarrollo regional, ni regulaciones comunes para impedir "la carrera hacia el fondo" de los salarios y las prestaciones sociales, ni una política común del medio ambiente, ni mucho menos implica un movimiento hacia instituciones políticas democráticas a escala continental $^{11}$.

\section{Los enemigos mexicanos del NAFTA}

El tratado de comercio también tiene enemigos en México. En general la oposición de izquierda al PRI ve en NAFTA una operación política para consolidar las reformas neoliberales que benefician a las grandes empresas multinacionales establecidas en México, y consolidar el poder decisorio del aparato tecnocrático del PRI, mientras que las posibles ganancias las ven limitadas por el hecho de darse en un contexto de falta de libertad y la mala distribución de la riqueza.

El comercio libre combinado con un gobiemo autoritario creará nuevas fuentes de riqueza, pero resultará en mayor concentración del ingreso y reforzará los aspectos autoritarios del sistema político mexicano. ... El comercio libre está de hecho minando las perspectivas de un cambio democrático en México ${ }^{12}$.

Si NAFTA resulta ser una alternativa a la apertura política y los demás cambios deseables en el sistema político mexicano, entonces el tratado significará un retroceso político. Y si las expectativas de beneficios no son tan positivas, existe el peli- gro de que las emergentes industrias mejicanas sean barridas por la competencia del comercio y de la inversión directa. El profesor Jorge Castañeda, uno de los más activos oponentes al NAFTA, lo pone asi: "En México dejar todo el mercado significa dar rienda suelta a los que lo dominan, los más poderosos y ricos".

Castañeda no se opone al proceso de integración de la economía mexicana con la estadounidense. El proceso comenzó hace cien años y su avance es inevitable. Pero antepone un acuerdo malo, que es el que se quiere llevar a cabo con un acuerdo bueno:

La elección está entre lo que se ha llamado un acuerdo exclusivamente comercial propio del capitalismo desregulado e individualista del mundo anglosajón y un acuerdo que iría más allá de los temas propiamente comerciales, que abarcara temas sociales y la relación entre el Estado y el mercado. El contraste entre estos dos acuerdos, el malo que tenemos y el bueno que queremos refleja la diferencia entre las dos clases de economías de mercado que existen en el mundo de hoy ${ }^{13}$.

El gobierno mexicano, por su parte, que ha apostado su caudal político a la carta de NAFTA está haciendo lo posible para enfrentar las críticas estadounidenses, a la vez que ignora o acalla las protestas internas. El 3 de octubre, el presidente Salinas anunció un plan para elevar los salarios de los obreros mexicanos, que supondrá al fisco un gasto de 6,000 millones de dólares. Estos aumentos de salarios los había prometido el presidente Salinas al presidente Clinton cuando se negociaron los acuerdos suplementarios ${ }^{14}$.

7. Los argumentos a favor: intereses económi$\cos$

Las grandes empresas multinacionales o globales están a favor de NAFTA. Es para ellas una cuestión de principio. La producción tiene que hacerse donde resulte más conveniente: el costo es naturalmente un elemento de la conveniencia, aunque no sea el único. Un acuerdo que fomenta la internalización de la producción no puede menos de serles aceptable aunque no todas deseen hacer 
uso de él. En un anuncio de una página entera del Washington Post pagada por el Citibank se podia leer.

Citibank cree que NAFTA es la clave del futuro éxito económico de Estados Unidos. Y esto es crucial para nosotros porque el éxito del Citibank está ligado al éxito de los trabajadores estadounidenses. En la medida en que crezca su prosperidad así crece la nuestra. Pero, grupos con intereses especiales se han alineado contra NAFTA amenazando seriamente su aprobación por el Congreso. Tu apoyo puede ser la diferencia. Llama a tu representante en el Congreso hoy mismo y hazle saber que piensas creer que NAFTA te favorece ${ }^{15}$.

En ediciones sucesivas de los principales periódicos de Estados Unidos, otras empresas han expresado en anuncios su apoyo al proyecto. El Chase Manhattan (los grandes bancos parece ser los que más empujan), los productores y exportadores de productos agrícolas y alimentos procesados (AG for NAFTA, una coalición de más de 175 grupos agrícolas), la industria pesada ( $G e$ neral Electric y $A B B$ ), American Textile Manufacturers Institute, empresas electrónicas (IBM, EDS Corporation), etc. Este entusiasmo de las multinacionales no quiere decir que todas las empresas estadounidenses se van a ir a México. En primer lugar, la inversión directa de las multinacionales es sobre todo en el primer mundo. Los defensores de NAFTA mencionan los casos recientes de la Mercedez Benz y la $B M W$ que van a establecer fábricas en Estados Unidos, pero no en México. La atracción de los salarios baratos tiene un límite. En segundo lugar, como el presidente Clinton no se cansa de repetir, el establecimiento de empresas en México, que tanto se teme, ya se puede hacer con facilidad. La General Electric, por ejemplo, una de las tres empresas más rentables de Estados Unidos está aumentando sus inversiones en India, China y México, y por las informaciones que tenemos, esto no tienen nada que ver con la formación de bloques comerciales, sino con el intento de servir a grandes mercados en rápida expansión ${ }^{16}$.

La oposición empresarial a NAFTA se da más en las pequeñas y medianas empresas, particularmente entre las que proveen a aquellos sectores que pueden desplazarse más a México, como el caso del automóvil, y las que no exportan. Sin embargo, son una voz insignificante, si se les compara tanto con otros opositores (los sindicatos y los ecologistas) como con otras empresas que defienden el acuerdo. Habria que hacer un análisis muy detallado de qué tipos de industria y de empresas están a favor o en contra. A veces es difícil determinar, por ejemplo, si la industria textil está a favor o en contra de NAFTA. Todo depende de quién hable por la "industria" textil".

Los eternos defensores del comercio libre, estén o no también a favor del GATT, apoyan a NAFTA. Aquí entra un conjunto de instituciones (think tanks, facultades de economía, escuelas de negocios, publicaciones, etc.) y personas (políticos, académicos y periodistas), llamados por sus adversarios "internacionalistas", aunque en el fondo defiendan los intereses de las empresas estadounidenses y la american way of life en una sociedad internacional muy interactiva, la aldea global $^{18}$. Algunos defienden a NAFTA más que por sus virtudes por los efectos negativos que tendria para la sociedad estadounidense y para el mundo entero un rechazo de los elementos positivos (apertura, solidaridad) que se pueden encontrar en el tratado para primar el nacionalismo, el aislacionismo, el estado de guerra comercial y otras actitudes y políticas por parte de Estados Unidos, que en el pasado han llevado no sólo a tensiones políticas y guerras, sino a grandes perjuicios para las economias nacionales de los paises industrializados. En palabras del escritor conservador George F. Will:

El Senador Bill Bradley exagera cuando compara la importancia de NAFTA con la compra de Louisiana, pero tiene razón cuando dice que probablemente es la medida más importante de

\section{NAFTA tiene de novedad el ser un experimento en integración de economías de muy distinto nivel de desarrollo.}


política exterior del mandato de Clinton, por el efecto que una derrota tendría sobre las políticas comerciales de todo el mundo, sobre las reformas internas de México y sobre los sentimientos nativistas y proteccionistas de este pais ${ }^{19}$.

Otros creen que el cierre, por lo menos relativo, de las fronteras comerciales de Estados Unidos es parte del programa que los enemigos de NAFTA, si triunfan, tratarian de vender a la opinión pública de Estados Unidos. Así lo pone el economista alemán del MIT, Rudiger Dombusch:

La coalición contra NAFTA tiene una agenda que sólo empieza aquí. El representante Richard Gephardt, Ross Perot, Pat Buchanan, Ralph Nader, los burócratas sindicales de Washington y numerosos grupos de benefactores están decididos a construir una gran muralla entorno al mercado estadounidense. Si ganan con una plataforma anti NAFTA, pasarán inmediatamente a poner de rodillas al $G A T T^{20}$.

\section{La polémica: ¿es para tanto?}

Tanto el libro de Ross Perot y Pat Choate, como el de Gary Hufbauer y Jeffrey Schott que le responden ${ }^{21}$, dan estimaciones sobre las pérdidas y ganancias netas que el tratado producirá en términos de puestos de trabajo. Si Hufbauer y Schott tuvieran razón, Estados Unidos ganaría 170,000 puestos de trabajo en los cinco años, pero si la tuvieran Perot y Choate, se perderían 480,000 . Pero la experiencia histórica en Estados Unidos da que esas pérdidas o ganancias se han tenido a veces en un solo mes. El pequerio número de puestos de trabajo ganados o perdidos por NAFTA no se notará en un océano de 130 millones de trabajadores estadounidenses.

El economista del MIT, Lester Thurow ha llamado la atención sobre las verdaderas proporciones de las consecuencias que se pronostican de uno y otro lado. Su mensaje es que NAFTA no supone un cambio revolucionario ${ }^{22}$. Thurow sentala que ya hay en el mundo muchos lugares con salarios bajos -entre otros Puerto Rico, que ya forma un mercado común con Estados Unidos-, donde cualquier empresa que quiera irse puede hacerlo sin ninguna dificultad. En cuanto a las estimaciones de pérdidas y ganancias de puestos de trabajo, nadie sabe exactamente cúntos se van a ganar o perder, por falta de modelos de predicción adecusdos. Lo que se puede saber con gran certeza, según Thurow, es que el impacto de NAFTA en la economía de Estados Unidos no va a ser muy considerable, porque la economía mexicana sólo es un 5 par ciento de ella, y Méxica no va a ser una locomotora que tire de la economia estadounidense.

Otro libro reciente Continental Shift ("Desplazamiento continental" $)^{23}$, pone la cuestión de los daños o beneficios en si la economía mexicana es complementaria o competitiva con la estadounidense. El problema, según Thurow, es que en el ámbito económico norteamericano hay una economía de primer mundo y una economia de tercer mundo. La economía mexicana es complementaria con la primera, pero competitiva con la segunda Aplicando este criterio se puede llegar a predecir

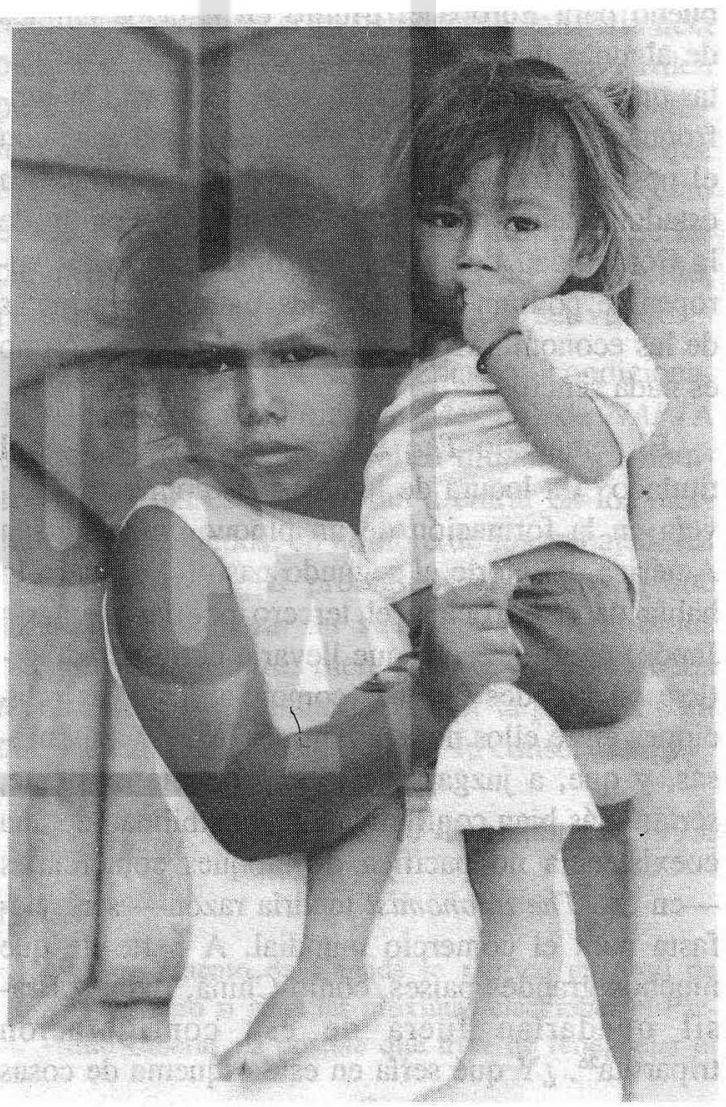


que la economía más desarrollada se beneficiará de la integración más estrecha con México, pero la parte subdesarrollada de la economía estadounidense se resentirá de la integración.

Al final, Lester Thrurow se decanta en favor de NAFTA como una cuestión de solidaridad y de buena educación:

Después de sentar a los canadienses a nuestra mesa económica y de que dos presidentes estadounidenses han extendido una invitación oficial a México para que se siente a comer con nosotros, retirar la invitación no es una posibilidad aceptable. Estaríamos confesando a nosotros mismos y al mundo que estamos dispuestos a tener un banquete económico con los canadienses, pero no con los mexicanos ${ }^{24}$.

\section{Lecciones para Europa y América Latina}

¿Qué está en juego para Europa en una polémica que parece tan lejana? En primer lugar, no sería bueno para Europa el triunfo en Estados Unidos de algunas de las fuerzas egocéntricas o centripetas que se oponen a NAFTA, que llevarian a la confrontación comercial con el exterior. Además con el posible fracaso del GATT por la intransigencia estadounidense con la política agrícola común de la Comunidad Económica Europea, y la crisis europea se podrían aumentar las fuerzas centrípetas de las economías industriales. Pero la situación no es nada sencilla.

El semanario The Economist, en un editorial titulado "La locura de los bloques comerciales"2s, veía en la formación de un bloque comercial en América del Norte el segundo paso (el primero lo había dado Europa y el tercero podria intentarlo Japón) en un proceso que llevaría al mundo a partirse en grandes bloques comerciales, cuyas relaciones entre ellos no tendrían por qué ser armoniosas, y que, a juzgar por la experiencia existente, serían más bien conflictivas. La posibilidad de una coexistencia no pacífica de bloques comerciales -en eso The Economist tendría razón- sería nefasta para el comercio mundial. A parte de que muchos grandes países, como China, India y Brasil quedarían fuera de esa configuración tripartita ${ }^{26}$. ¿Y qué sería en este esquema de cosas la suerte de los países más pobres de Africa?

Por lo tanto, un triunfo de NAFTA junto al fracaso de la ronda Uruguay del GATT podría ser negativo para Europa y el mundo, pero un fracaso del NAFTA y del GATT conjuntamente sería sin duda mucho peor. Para los intereses particulares de Europa, lo mejor sería un triunfo del GATT y un fracaso de NAFTA (ya lo dice Lee A. Jacocca en sus anuncios televisivos a favor de NAFTA: "El voto contra NAFTA es una decisión que favorece al Japón y a Europa"), aunque, por supuesto, el punto de vista europeo no debe ser el punto de vista de la solidaridad intemacional.

NAFTA tiene de novedad el ser un experimento en integración de economías de muy distinto nivel de desarrollo. La operación es en sí misma difícil y compleja por los muchos aspectos que tiene, pero es importante para articular un orden económico no excluyente. Es un experimento nuevo de integración norte-sur que, aparte de los problemas políticos de México, ha creado esperanza e ilusión en otros países de América Latina, como una nueva forma, más igualitaria y equilibrada, de aquel panamericanismo, que distorsionado por la guerra fría se convirtió en un instrumento de dominación y de intervención de Estados Unidos en los asuntos internos del continente americano.

Los líderes latinoamericanos, en la medida que reflejan los intereses de sus respectivos pueblos, han formulado esta vieja aspiración del continente de diversas maneras. El presidente de Chile, Patricio Alwyin, que espera en la antesala de NAFTA para negociar su adhesión al tratado, decía:

NAFTA representa un anhelo para la región y un signo de la política que Washington debe seguir con América Latina.

El presidente colombiano, César Gaviria, por su parte afirmaba:

El NAFTA será un primer paso en la configuración de una gran zona libre de comercio en el hemisferio americano.

Y finalmente el presidente Salinas, uno de los protagonistas del uratado:

La aprobación del Tratado de libre comercio 
mostraría si Estados Unidos está realmente decidido a tener una relación diferente con América Latina ${ }^{27}$.

Tengo la impresión de que estas afirmaciones reflejan la opinión más extendida entre los latinoamericanos.

\section{Conclusiones}

¿Dónde debemos colocamos ante este debate la gente solidaria? La verdad es que tanto la nueva izquierda estadounidense (en la cual no cuento a los sindicatos) como la izquierda mexicana y los ecologistas de ambos países están en contra del tratado como ahora existe. Ya vimos que esto no parece ser una oposición en principio a la integración de México con sus ricos vecinos del norte (la de los sindicatos americanos sí lo es). La izquierda de los países implicados ve a NAFTA como una consolidación de tendencias que ellos combaten en sus términos de referencia específicos. Por un lado, se oponen a una internacionalización de las empresas que no tiene ninguna consideración con lo que resta de las economías nacionales, y por otro, combaten la exposición al mercado internacional (a través de la competencia entre trabajadores de diversos paises) de los sistemas de protección social resultado de las luchas sociales de tiempos pasados.

La perspectiva de estas izquierdas locales, sin embargo, puede no considerar la necesidad de una organización de la economía mundial que beneficie a los países más pobres, a los emergentes y a los que se van desarrollando a costa de grandes sacrificios, que sólo se justificarán a posteriori si sus economías llegan a desarrollarse económica y socialmente. Para este desarrollo global hacen falta mercados abiertos, hace falta que los productos de los países en desarrollo, cualquiera que sea su estadio de evolución, lleguen sin obstáculos a los mercados opulentos de los países ricos (unilateralmente, por supuesto). Para esto se necesita un sistema de comercio abierto, multilateral, no discriminatorio (aunque asimétrico para compensar las desigualdades de partida), con reglas de conducta explícitas comúnmente aceptadas y obedecidas. Esto implica para los países ricos comer- ciar con países que hoy por hoy tienen salarios más bajos, menores cotas de protección social y medidas más tolerantes de protección ambiental.

A esto aspiramos los que desde la perspectiva de las instituciones existentes, como el GATT, queremos reformar las actuales reglas para tener en cuenta las necesidades de los países más pobres. El ideal seria una ronda Uruguay generosa y amplia que tuviera éxito antes de terminar el año. A falta de estos arreglos generales, los arreglos regionales y parciales, siempre que no sean claramente nocivos para los paises participantes, podrían ofrecer una altemativa realista y duradera a un acuerdo general sobre comercio e inversión. En cualquier acuerdo sobre comercio en que se reduce la protección a algunos sectores productivos siempre se perjudica a los que viven de las actividades productivas en los sectores protegidos, aunque normalmente se beneficia a muchos más, es decir, a los que consumen los productos y servicios, que son muchos más. Se suele dar como una regla para constatar si ha habido una ganancia neta para el conjunto de la sociedad, si los que pierden pueden ser compensados por los que ganan de manera que ninguno de aquéllos quede peor de lo que estaba antes y éstos queden mejor que antes. Esto es en teoría, claro está, porque el problema de las compensaciones a los perdedores no es sencillo, pero como criterio de bienestar puede ser válido.

Por eso abogo por una aceptación condicionada (a la reforma política en México) de NAFTA por parte de la opinión pública mundial, en nombre de los países que aspiran a entrar en los mercados desarrollados, en nombre de los trabajadores mal pagados y mal protegidos socialmente que sólo podrán mejorar su suerte si les permitimos integrarse en nuestros mercados, en nombre de los medios ambientes maltratados por la pobreza para que sea económicamente viable su conservación.

Washington, 19 de octubre de 1993.

\section{Notas}

1. El parlamento de Canadá lo ratificó en abril de 1993. Pero si gana las próximas elecciones el Partido Liberal, es posible que trate de renegociar el 
acuerdo.

2. Un libro reciente de autores críticos de NAFTA en los tres países miembros ha destacado los aspectos políticos del tratado: Ricardo Grinspun y Maxwell Cameron (ed.), The political Economy of North American Free Trade, New Tork: St. Martins Press, 1993.

3. Como en la mayor parte de los países de América Latina, el proteccionismo y la orientación hacia el mercado intemo han caracterizado la política comercial de México durante varias décadas. Pero a principios de 1985 y como parte de un plan global de ajuste económico, impuesto en parte por la necesidad de renegociar la deuda externa, México se embarco en un ambicioso programa de liberalización comercial. Los aranceles se redujeron a la mitad y la cobertura de las licencias de importación se redujo del 92 al 20 por ciento.

4. Estos análisis son frecuentes en la prensa cotidiana estadounidense. Un buen ejemplo de ellos lo offece: Mary McGrory, "The NAFTA Disasta", The Washington Post, 10 de octubre de 1993, p. C-1 y C-5.

5. Ross Perot y Pat Choate, Save your job, save our country. Why NAFTA must be stopped - Now, New York: Hyperion Press, 1993. Un libro que el periodista J. W. Anderson ha llamado "Perot's little book of NAFTA nonsense".

6. Veinticinco asociaciones ecologistas y de protección de animales, que van del Sierra Club y Friends of the Earth a la Doris Day Animal League, han publicado un manifiesto " 8 Fatal Flawas of NAFTA. Environmental Catastrophe, Canada to México", The Washington Post, 22 de septiembre de 1993, p. A-29.

7. Representante (Ministro) de Comercio de Estados Unidos, Mickey Kantor, "NAFTA maintains U.S. Environment Standards", Letter to the Editor, New York Times, 23 de septiembre de 1993.

8. "Labor and NAFTA", The Washington Post (editorial), 23 de septiembre de 1993, p. A-24.

9. The Washington Post, loc. cit.

10. "Kirkland: No compromise on NAFTA", The Washington Post, 1 de septiembre de 1993, p. F-3. Lane Kirkland, presidente de la federación sindical $A F L$ (American Federation of Labor)-CIO excluy6 cualquier compromiso con la Casa Blanca sobre el Acuerdo norteamericano de libre comercio NAFTA, y avisó que los sindicatos van a quemar hasta el último cartucho para derrotar el tratado en el Congreso.

11. Robert Kutner, "Foreword", en R. Grispun M. A. Cameron, The Political Economy of North
American Free Trade, New York: St Martin s Press, 1993, p. XIV.

12. Adolfo Aguilar Zinser, "Authoritariasm and North American Free Trade: The Debate in Mexico", Capímulo 13, en Ricardo Grinspun y Maxwell Cameron, The Political Economy of North American Free Trade, New York: St. Martin \& Press, 1993, pp. 205-216.

13. Jorge G. Castañeda, "Another NAFTA what a good agreement should offer", Capítulo 6 en Ralph Nader, William Greider y otros The Case Against Free Trade, New York: North Atlantic Books, 1993, pp. 80-85.

14. The New York Tines, 4 de octubre de 1993, p. D1.

15. The Washington Past, 24 de octubre de 1993, p. A-40.

16. Ver "GE'S Brave New World", Business Week, 8 de noviembre de 1993, pp. 64-67.

17. Carta de Walter $Y$. Elisha, director general de Springs Industries Inc.: "NAFTA ofrece a la industria textil de Estados Unidos una de las mejores oportunidades en nuestra memoria. Puede caracterizar a la industria como una muy fuerte partidaria", Readers Repport. Business Week, 18 de octubre de 1993, p. 10. No todos los empresarios textiles piensan asf.

18. Un ejemplo es el estudio de la Brookings Institution: Nora Lustig, Barry P. Bosworth y Rober Z. Lawrence (ed.), North American Free Trade, Assesing the Impact, Washington: The Brookings Institution, 1992. Las estimaciones cuantitativas del estudio son todas muy favorables a NAFTA.

19. George F. Will, "NAFTA and The Great Wall of Fear", The Washington Post, 26 de septiembre de 1993, p. C-7.

20. Rudi Dombusch, "Workers will lose the most if NAFTA dies", Business Week, 8 de noviembre de 1993, p. 20.

21. Gary Clide Hufbauer y Jeffrey J. Schoth NAFTA: an Assessment, Washington: Institute for International Economics, 1993.

22. Lesteer Thurow, "An American Common Market", The Washington Post, 31 de octubre de 1993 (Book World).

23. William A. Orme Jr., Continental Shift. Free Trade and the New North America, Washington: The Washington Post Company, 1993.

24. Lester Thurow, loc. cit.

25. The Economist, "Trade Block Folly" (editorial), 20 de abril de 1991.

26. Lo negativo de la formación de un segundo bloque comercial se ponía en que este paso se daba preci- 
samente cuando las negociaciones para reforzar el sistema abierto y multilateral de comercio que propugna el GATT en el marco de la ronda Uruguay estaban en franca crisis. Sin embargo, el gobiemo norteamericano continúa afurmando que la conclusión de la ronda Uruguay es la primera prioridad de su política comercial y que el NAFTA llevará a fortalecer el GATT más que a debilitarlo. Habrá que ver si la formación de bloques conducirá inexorablemente a menos apertura y libertad en los intercambios internacionales, como predicen The
Economist y defensores del GATT como Jagdish Bhagwati (The World Trading System at Risk), o a una gradual aproximación al ideal del comercio libre mundial. El premio nóbel Maurice Allais piensa que este es un único camino viable hacia el comercio libre.

27. Las citas de los presidentes latinoamericanos están tomadas del artículo de Albert Muller, "El NAFTA: un anhelo de solidaridad", Diario de las Américas, 26 de octubre de 1993, p. 5-A. 